\title{
The Safety and Complications of "Z-entry" Technique of Trocar Insertion in Laparoscopic Gynecological Surgery
}

\section{ARTICLE INFO}

\section{Article Type}

Original article

Authors

AboTaleb Saremi ${ }^{1 *}$ iD, MD

Mohammad Reza Nateghi ${ }^{1}$, iD MD

Sarem Fertility \& Infertility Research Center (SAFIR) \& Sarem Cell Research Center (SCRC), Sarem Women's Hospital, Iran University of Medical Sciences (IUMS), Tehran, Iran.

\section{*Corresponding Author}

Address: Sarem Women Hospital, Basij Square, Phase 3, EkbatanTown, Tehran, Iran. Postal code: 1396956111

Phone: +98 (21) 44670888

Fax: +98 (21) 44670432

Dr.saremi@sarem.org

\section{Article History}

Received: February 15, 2021

Accepted: March 08, 2021

e Published: September 29, 2021

\section{ABSTRACT}

Background and Aims: Laparoscopy is a minimally invasive surgical technique for abdominal and gynecological surgeries. To access the internal organs through the abdominal wall, a special tool called a trocar is used. The trocar enters the abdomen using a variety of techniques. The aim of this study was to evaluate the safety and possible complications of trocar insertion into the abdomen for gynecological laparoscopy using the "Z-entry" method.

Methods: From March 2018 to March 2020, 435 patients underwent laparoscopic gynecological surgery in Sarem specialized hospital. The consequences of trocar entering the abdomen with the "Z-entry" technique, including visceral injury, vascular injury, umbilical hernia, hematoma, massive hemorrhage, infection, and mortality induced by trocar insertion, were evaluated. The results were analyzed and reported in the form of descriptive statistics and frequency distribution tables with SPSS statistical software.

Findings: This study showed that the rate of complications and problems due to insertion of trocar by "Z-entry" method was very low in these patients. They were comprised of one case of infection $(0.2 \%)$, one case of hematoma $(0.2 \%)$, one case of trocar entry into blood vessels $(0.2 \%)$ and two cases $(0.4 \%)$ of trocar entry into the viscera. However, no case of umbilical hernia, massive hemorrhage, and death induced by trocar insertion was found.

Conclusion: Insertion of trocar by the "Z-entry" method in laparoscopic gynecological surgeries is associated with minimal problems and complications and therefore this technique can be used as a safe method to insert trocar into the abdomen in laparoscopic surgeries.

Keywords: Laparoscopic Gynecological Surgery; Trocar Insertion; "Z-entry" Technique; Safety; Complications.

Copyright(C) 2021, ASP Ins. This open-access article is published under the terms of the Creative Commons Attribution-Noncommercial 4.0 International License which permits Share (copy and distribute the material in any medium or format) and Adapt (remix, transform, and build 


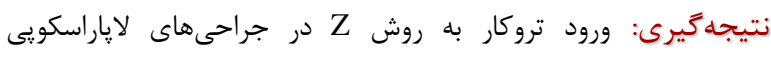

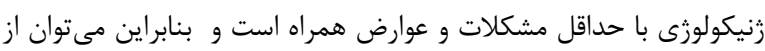

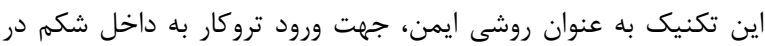
جراحىهاى لإياراسكويى بهره ترفت.

كليد وارثها: جراحى لاياراسكويى زنيكولوزى؛ تكنيك ورود تروكار؛ روش

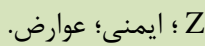

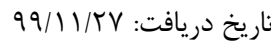

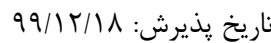

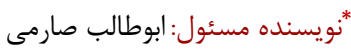

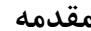

براى نخستين بار در سال • ل191، هانس كريستين جاكوبز 'اولين عمل

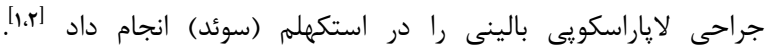

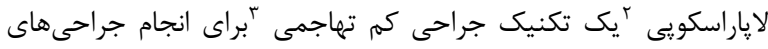

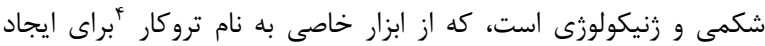

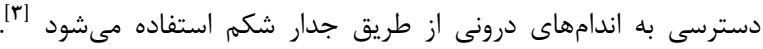

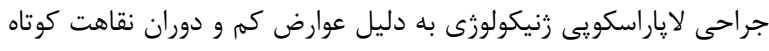

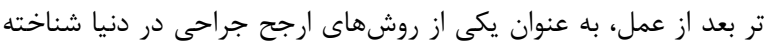

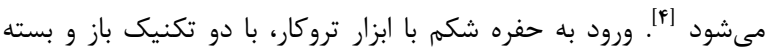

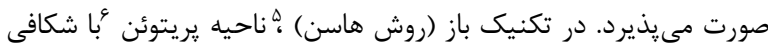

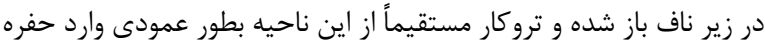

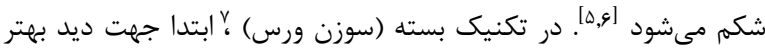

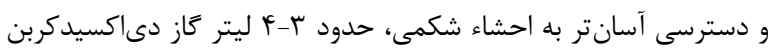

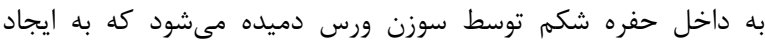

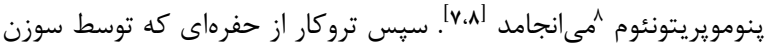

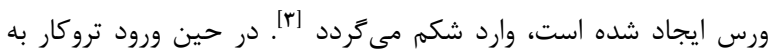

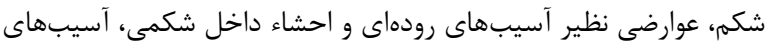

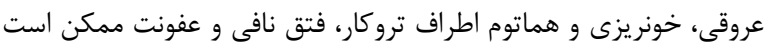

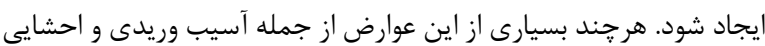

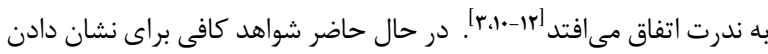

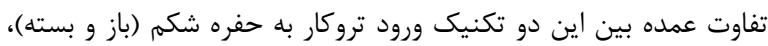

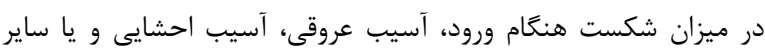

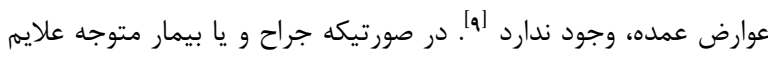

Hasson Technique ${ }^{\circ}$ Peritoneum

Veress Needle Pneumoperitoneum

\section{ايمنى و عوارض ورود تروكار به داخل شكم به روش Z Z در جراحى

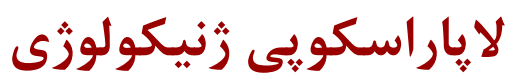

ابوطالب صارمى * (iD)، محمد رضا ناطقى (iD) ' مركز تحقيقات بارورى و نابارورى صارم، يزوهشكده سلولى و مولكولى و

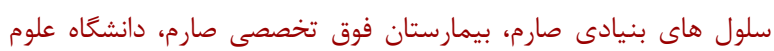
يزشكى ايران، تهران، ايران.

جكيده

اهداف: لاياراسكويى يك تكنيك جراحى كم تهاجمى براى انجام جراحى

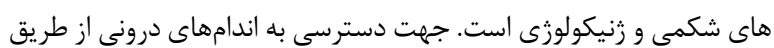

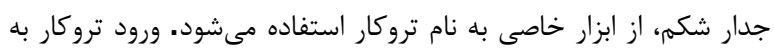

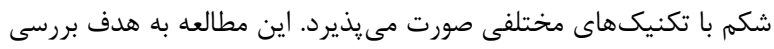

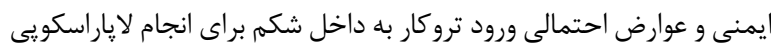

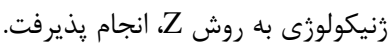

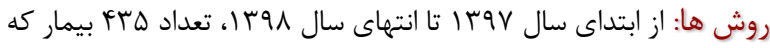

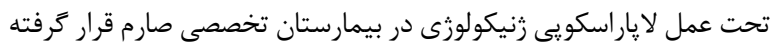

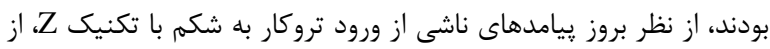

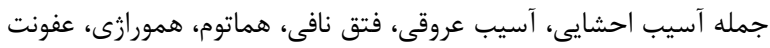

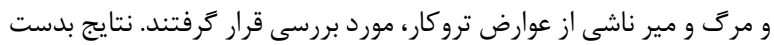

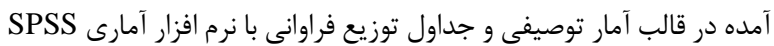

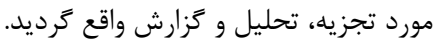

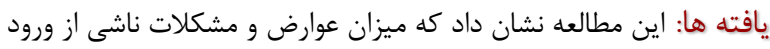

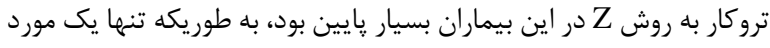

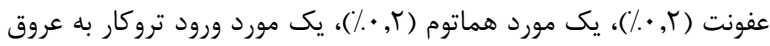

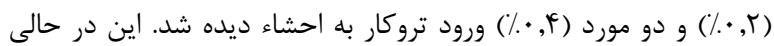

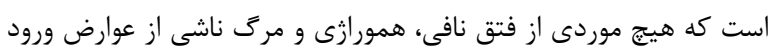
تروكار يافت نشد.

Hans-Christian Jacobaeus (1879-1937) Laparoscopy Minimally Invasive Surgery (MIS) Trocar 

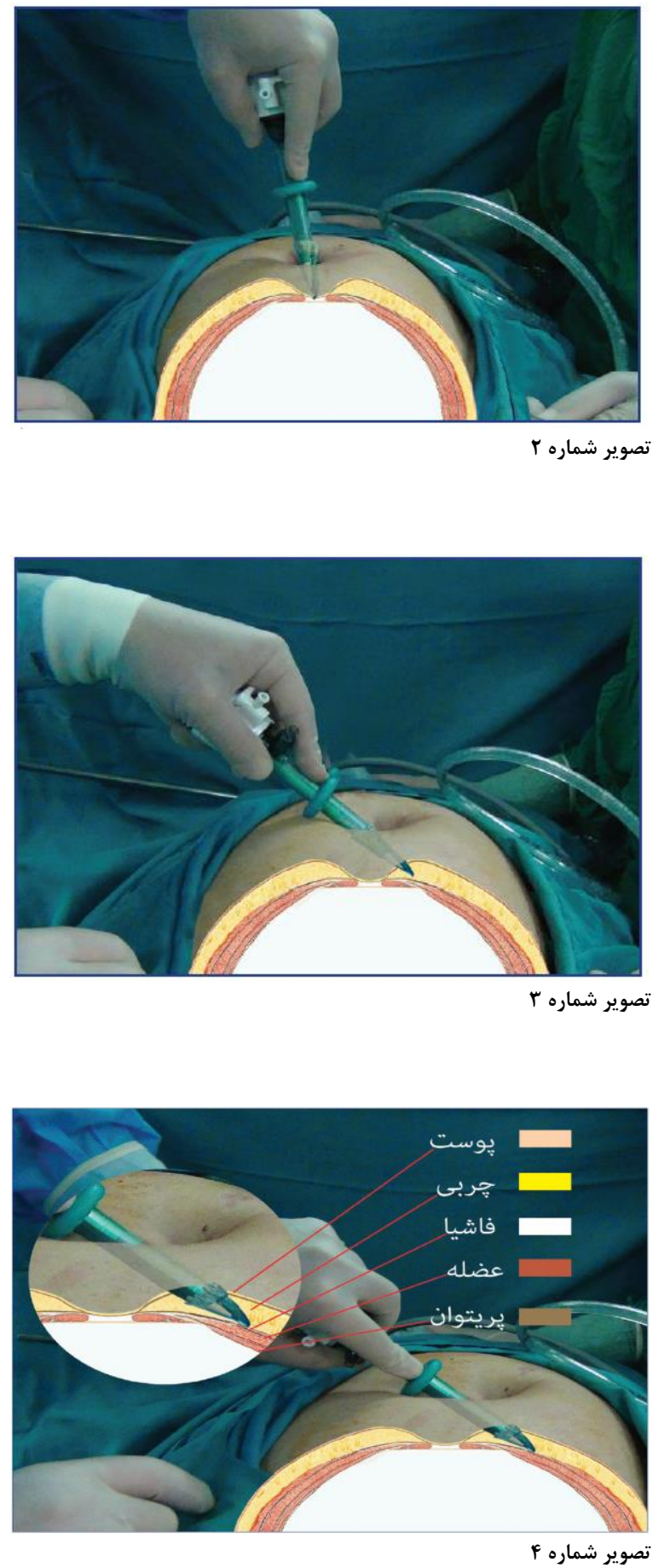

Mandarin '" Rectus Abdominis Muscle
زودرس عوارض نكرديده و براى درمان به موقع آن اقدام نشود، مشكلات

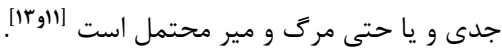

\section{ورود تروكار به داخل شكم به روشج:}

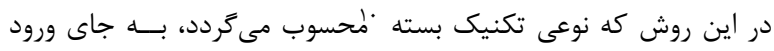

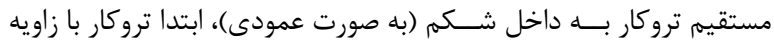

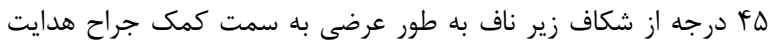

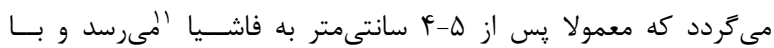

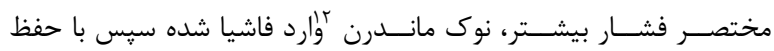

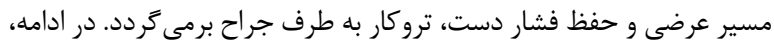

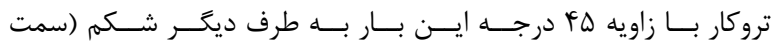

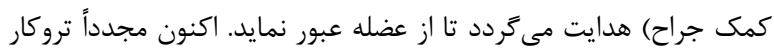

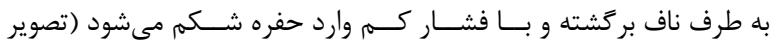

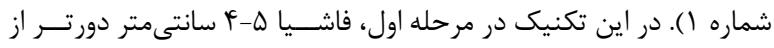

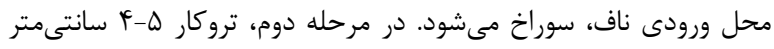

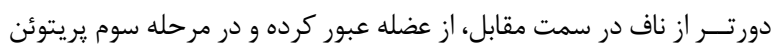

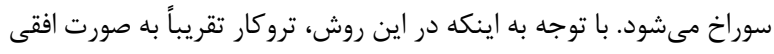

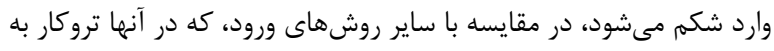

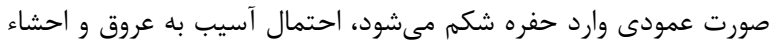

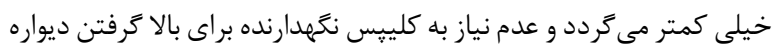

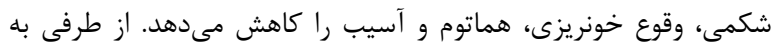

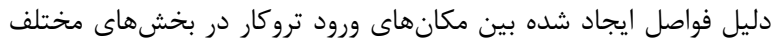

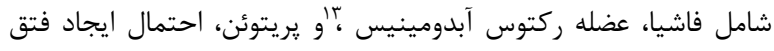

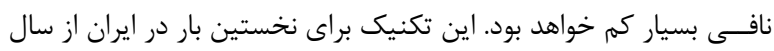

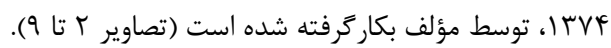

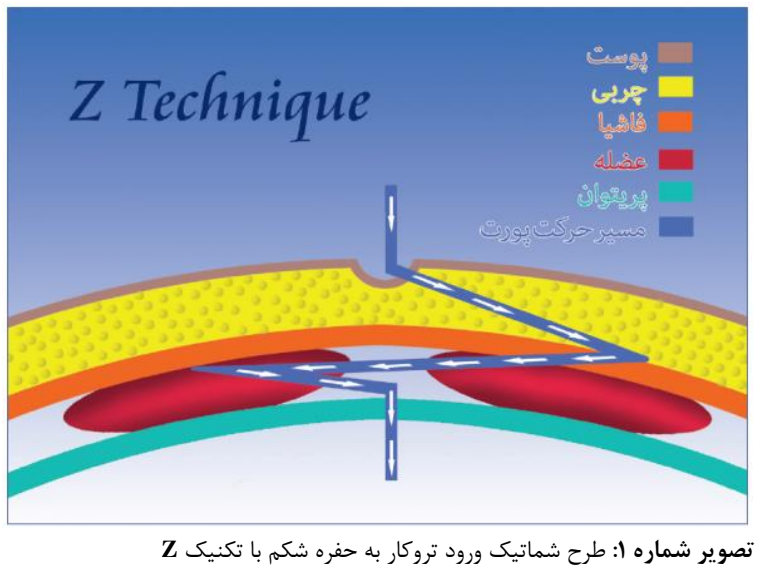

Z-Entry Technique
Close Technique
Fascia

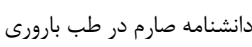




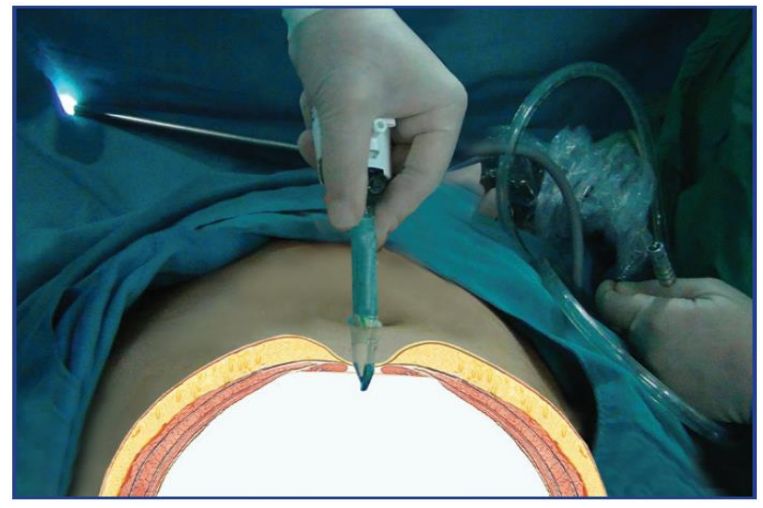

تصوير شماره

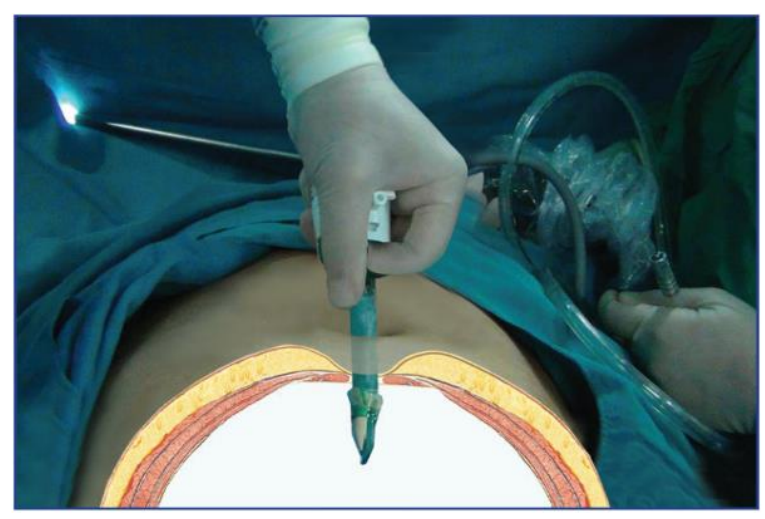

9 تصوير شماره

اين مطالعه به هدف بررسى ايمنى و عوارض احتمالى ورود تروكار به داخل

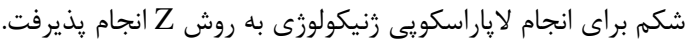

\section{روش ها}

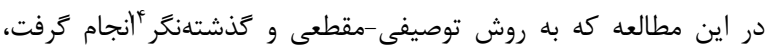

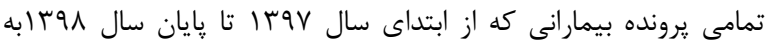

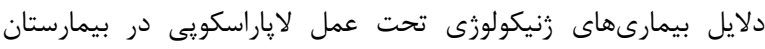

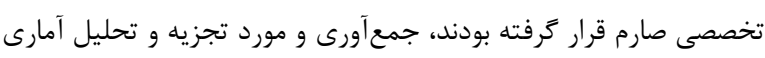

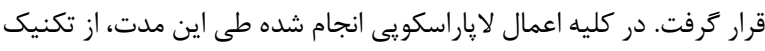

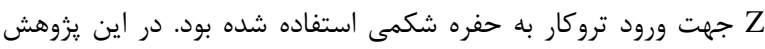

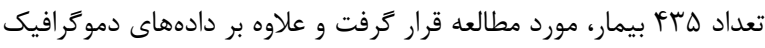

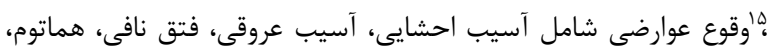

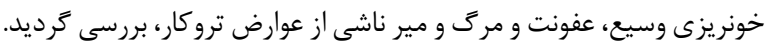

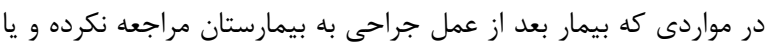

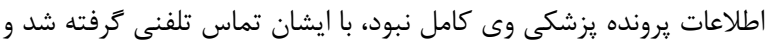
اكر عوارضى جون تورم و تغيير شكل اطراف ناف راذ اذكر مىكرد، بيمار مجدداً

Demographic Data ${ }^{10}$

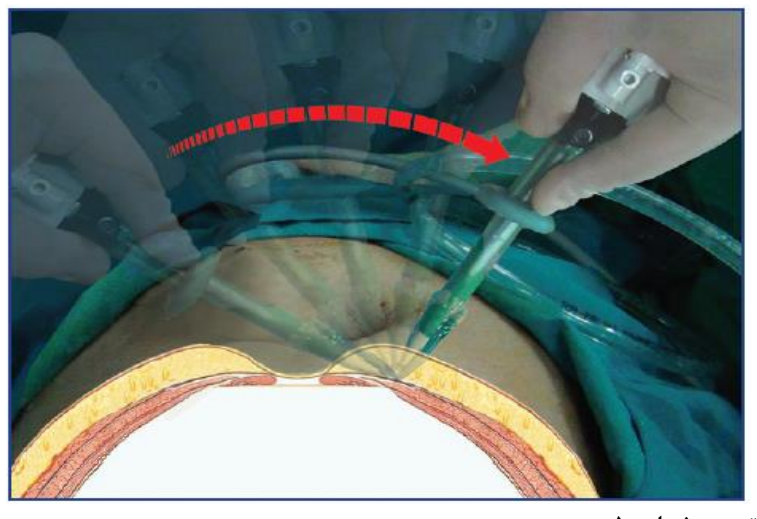

تصوير شماره ها

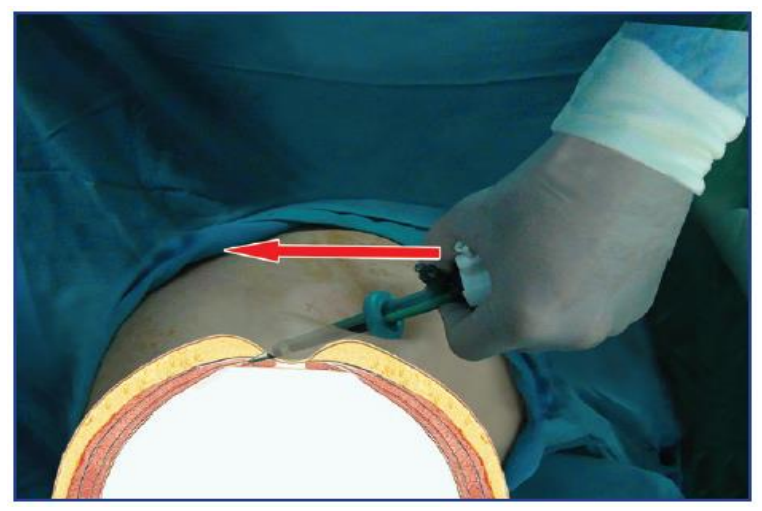

تصوير شماره

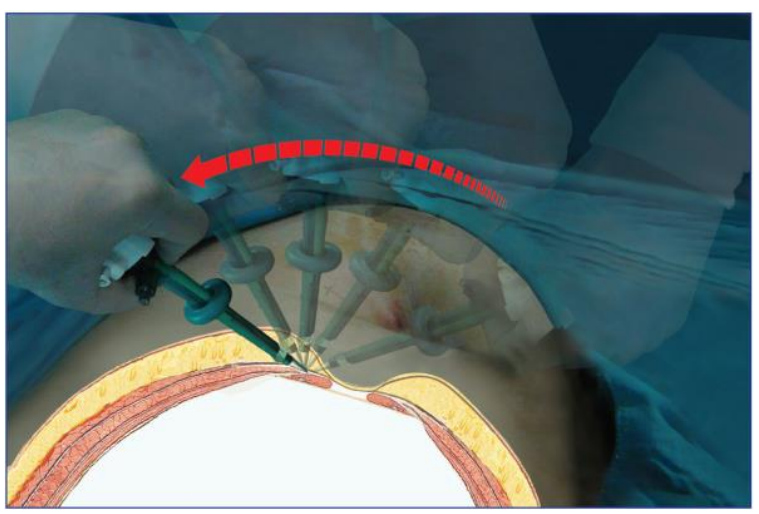

V تصوير شماره
Retrospective Cross-Sectional Study '"

دانشنامه صارم در طب بارورى 


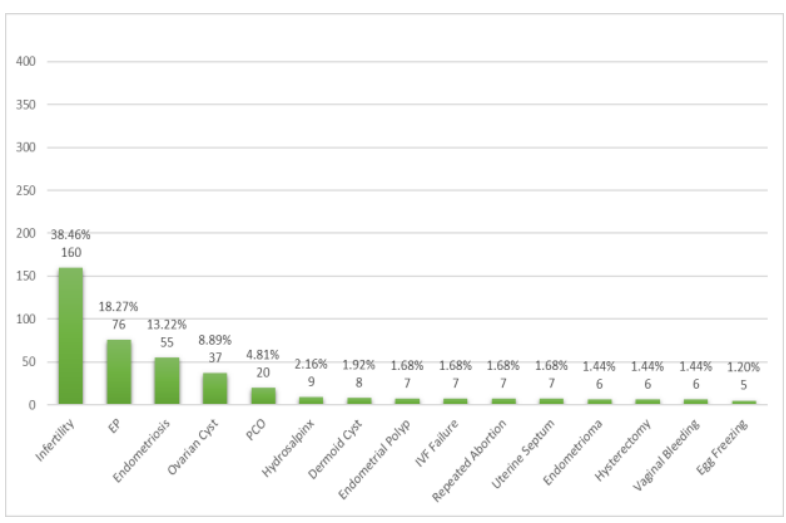

نمودار ا: علل عمده جراحى لإياراسكويى در افراد مورد مطالعه

در اين مطالعه دو ساله، ميزان عوارض و مشكلات ناشى از ورود تروكار به

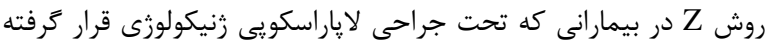

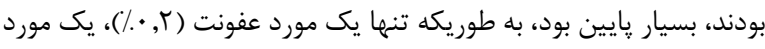

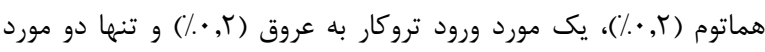

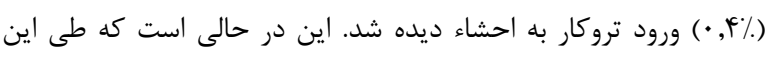

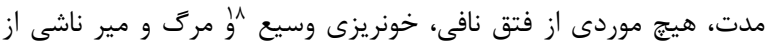

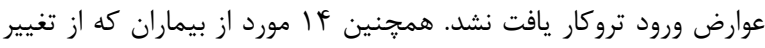
شكل اطراف ناف شكايت داشتند، طى بررسى در در مراجعه حضورى و و معاينه نئن

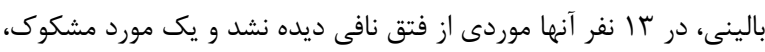

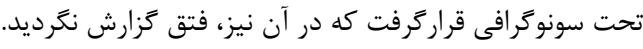

\begin{tabular}{|c|c|c|}
\hline درصد & فراوانى & عارضه \\
\hline \multicolumn{3}{|r|}{ ورود تروكار به احشاء } \\
\hline$\cdot,+4$ & $r$ & بله \\
\hline$१ १, \Delta F$ & Frr & خير \\
\hline \multicolumn{3}{|r|}{ ورود تروكار به عروق } \\
\hline • & 1 & بله \\
\hline ११,マV & Fry & خير \\
\hline \multicolumn{3}{|r|}{ هماتوم } \\
\hline • & 1 & بله \\
\hline १९, V & fry & 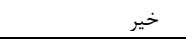 \\
\hline \multicolumn{3}{|r|}{ عفونت } \\
\hline • & 1 & بله \\
\hline १९,४७ & fre & خير \\
\hline \multicolumn{3}{|r|}{ فتق نافى } \\
\hline . & . & بله \\
\hline $1 \ldots$ & fro & خير \\
\hline \multicolumn{3}{|r|}{ همورازى } \\
\hline$\cdot$ & . & بله \\
\hline $1 \cdots$ & fro & خير \\
\hline \multicolumn{3}{|c|}{ مركى ناشى از عوارض ورود تروكار } \\
\hline$\cdot$ & $\cdot$ & بله \\
\hline $1 \cdots$ & fro & 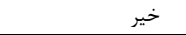 \\
\hline
\end{tabular}

Massive Hemorrhage '
معاينه شده و در صورت شك به وجود فتق نافى، تحت بررسى سونوگرافى

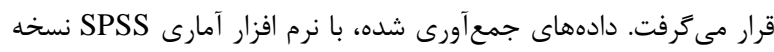

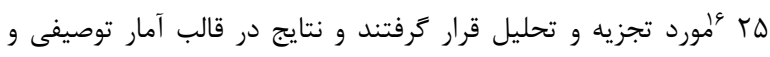

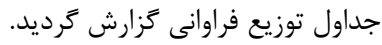

\section{نتايج}

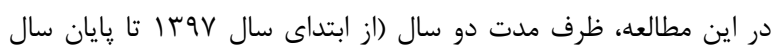

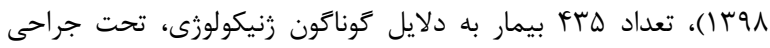

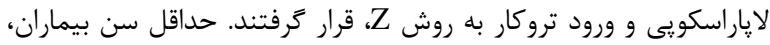

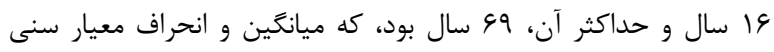

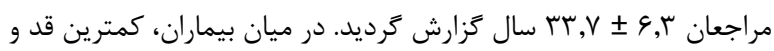

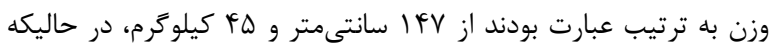

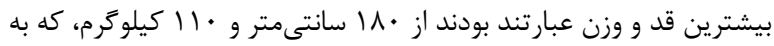

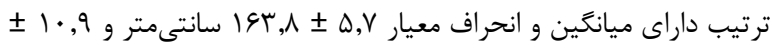

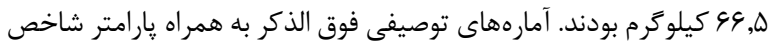

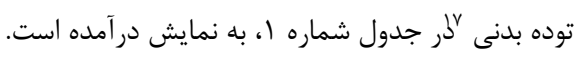

جدول ا: مشخصات دموكرافيك افراد مورد مطالعه

\begin{tabular}{|c|c|c|c|}
\hline حداكثر & حداقل & ميانكين \ انحراف معيار & بارامتر ( FrA نفر) \\
\hline 99 & 19 & $r r, v \pm \varepsilon, r$ & سن (سال ) \\
\hline $1 \Lambda$. & IfV & $\mid \varepsilon r, \Lambda \pm \Delta, V$ & قد (سانتى,متر) \\
\hline 11. & FQ & $94, \Delta \pm 1 \cdot, 9$ & وزن (كيلوكرم) \\
\hline$r \wedge, q$ & 19,0 & $r f, v \pm r, q$ & شترمربع) توده بدنى (كيلوكرم بر \\
\hline
\end{tabular}

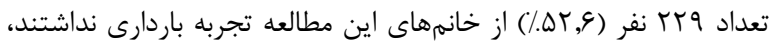

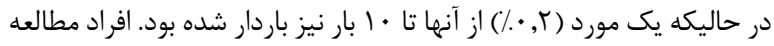

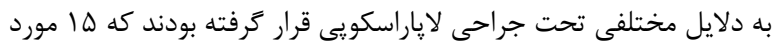

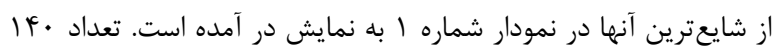

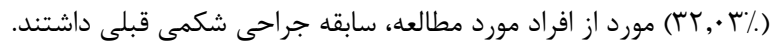

IBM SPSS, Version 25 (IBM Corp., Armonk, N.Y., USA) ' Body Mass Index (BMI) ''

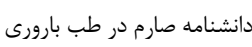


روشهاى ديخر داراى مزايا و ارجحيت بالاترى است و مىتوان از آن به

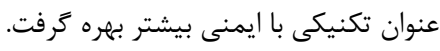

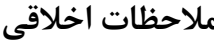

كليه دادههاى هويتى بيماران در اين مطالعه، محرمانه و محفوظ مى باشيد.

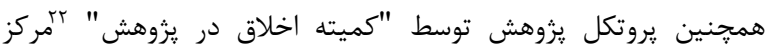
تحقيقات بارورى و نابارورى صارم تأييد كرديد.

\section{تشكر و قدردانى}

از لطف بى دريغ كاركنان محترم بيمارستان تخصصى صارم و مركز تحقيقات

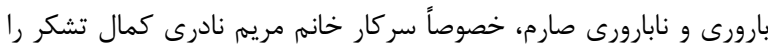

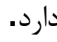

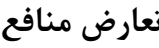

در اين مطالعه تعارض منافع وجود نداشت.

هزينههاى اين يروهش توسط مركز تحقيقات بارورى و نابارورى صارم تأمين

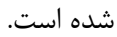

1.

Hatzinger M, Häcker A, Langbein S, Kwon S, Hoang-Böhm J, Alken P. Hans-Christian Jacobaeus (1879-1937). Der Urol 2006459 [Internet]. 2006 [cited 2021 Sep 23];45(9):1184-6. Available from: https://link.springer.com/article/10.1007/s00 120-006-1069-8

2. Hatzinger M, Kwon ST, Langbein S, Kamp S, Häcker A, Alken P. Hans Christian Jacobaeus: Inventor of Human Laparoscopy and Thoracoscopy.

https://home.liebertpub.com/end [Internet]. 2006 Dec 4 [cited 2021 Sep 23];20(11):848-50. Available from: https://www.liebertpub.com/doi/abs/10.1089 /end.2006.20.848

3.

Vilos GA, Ternamian A, Dempster J, Laberge PY, Vilos G, Lefebvre G, et al. Laparoscopic Entry: A Review of Techniques, Technologies, and Complications. J Obstet
در طول دو دهه كذشته، در تكنيك جراحى لاياراسكويى به دليل ايمنى

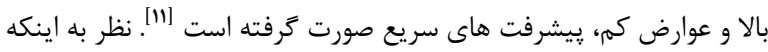

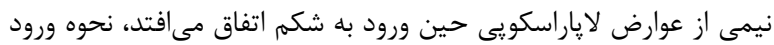

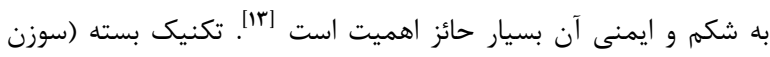

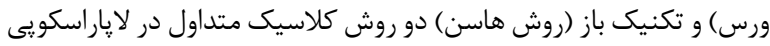

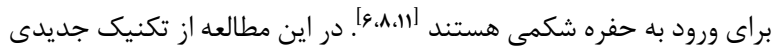

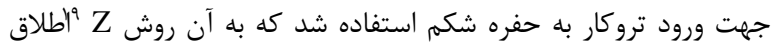

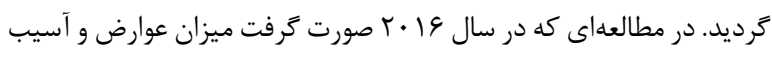

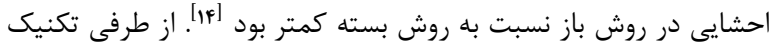

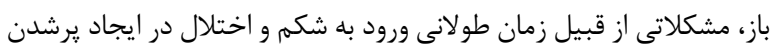

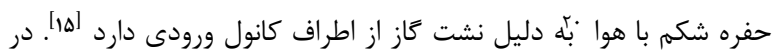

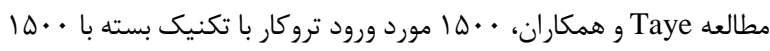

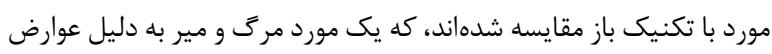

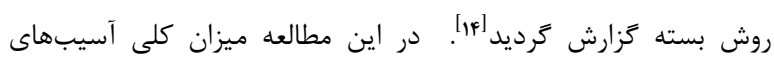

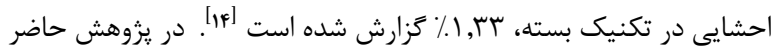

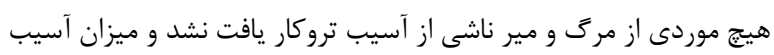

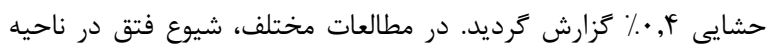

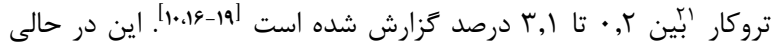

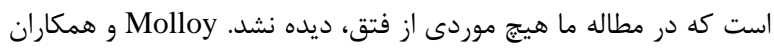

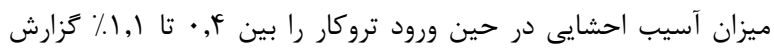

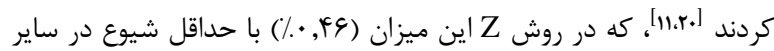

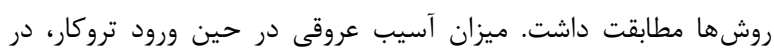

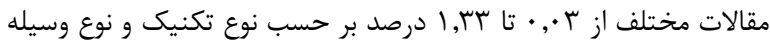

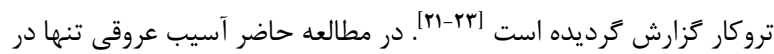

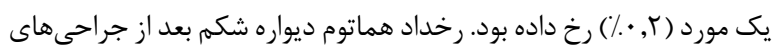

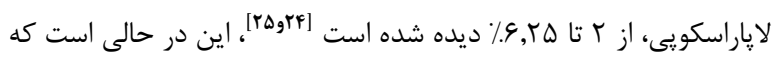

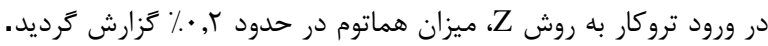

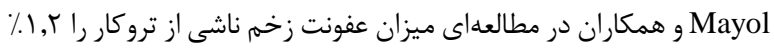

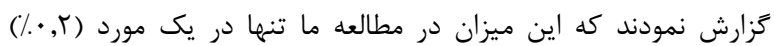

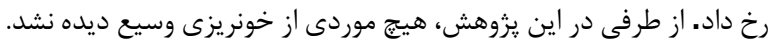

\section{نتيجه حَيرى}

امروزه جراحى لايار اسكويى به طور وسيع و گستردهاى در جراحى هاى شكم

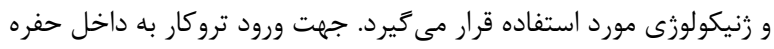

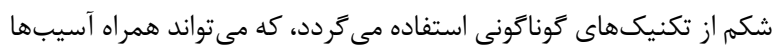

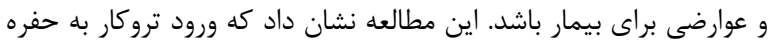

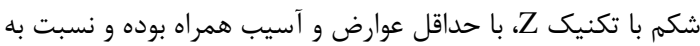

Z-Entry Technique Insufflation

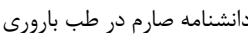


College of Obstetricians and Gynaecologists; 2002 [cited 2020 Dec 24]. p. 246-54. Available from:

https://obgyn.onlinelibrary.wiley.com/doi/full /10.1111/j.0004-8666.2002.00246.x

12.

Gadekar A, Rege J, Satia M, Hambarde S. Omental herniation through trocar site. Ann Afr Med [Internet]. 2012 Jul [cited 2020 Dec 24];11(3):191. Available from: http://www.annalsafrmed.org/text.asp?2012/ 11/3/191/96885

13. Mintz M. Risks and prophylaxis in laparoscopy: a survey of 100,000 cases. J Reprod Med. 1977;18(5):269-72.

14. Taye MK, Fazal SA, Pegu D, Saikia D. Open versus closed laparoscopy: yet an unresolved controversy. J Clin diagnostic Res JCDR. 2016;10(2):QC04.

$15 . \quad$ Bonjer HJ, Hazebroek EJ, Kazemier G, Giuffrida MC, Meijer WS, Lance JF. Open versus closed establishment of pneumoperitoneum in laparoscopic surgery. Br J Surg [Internet]. 1997 May 1 [cited 2021 Sep 21];84(5):599-602. Available from: https://onlinelibrary.wiley.com/doi/full/10.10 46/j.1365-2168.1997.d01-1355.x

16. Crocetti D, Sapienza P, Pedulla` G, De Toma G. Reducing the risk of trocar site hernias. Ann R Coll Surg Engl [Internet]. 2014 [cited 2020 Dec 24];96(7):558. Available from: https://www.ncbi.nlm.nih.gov/pmc/articles/P MC4473458/

17. Swank HA, Mulder IM, la Chapelle CF, Reitsma JB, Lange JF, Bemelman WA. Systematic review of trocar-site hernia. Br J Surg [Internet]. 2012 Jan 30 [cited 2021 Sep 23];99(3):315-23. Available from: https://academic.oup.com/bjs/article/99/3/3 $15 / 6138733$

18. Tonouchi H, Ohmori Y, Kobayashi M, Kusunoki M. Trocar Site Hernia. Arch Surg [Internet]. 2004 Nov 1 [cited 2021 Sep 23];139(11):1248-56. Available from: https://jamanetwork.com/journals/jamasurge ry/fullarticle/397584

19. Nacef K, Chaouch MA, Chaouch A, Ben Khalifa M, Ghannouchi M, Boudokhane M. Trocar site post incisional hernia: About 19 cases. Pan Afr Med J [Internet]. 2018 [cited 2020 Dec 24];29. Available from: https://pubmed.ncbi.nlm.nih.gov/30061961/
Gynaecol Canada [Internet]. 2007 May [cited 2020 Dec 24];29(5):433-47. Available from: https://pubmed.ncbi.nlm.nih.gov/17493376/

4. Chang WC, Huang SC, Sheu BC. Advances in Gynecological Laparoscopic Surgery. J Formos Med Assoc. 2010 Apr 1;109(4):245-7.

5. Günenç MZ, Yesildaglar N, Bingöl B, Önalan G, Tabak S, Gökmen B. The safety and efficacy of direct trocar insertion with elevation of the rectus sheath instead of the skin for pneumoperitoneum. Surg Laparosc Endosc Percutaneous Tech [Internet]. 2005 [cited 2020 Dec 24];15(2):80-1. Available from: https://pubmed.ncbi.nlm.nih.gov/15821619/

6. Hasson HM. A modified instrument and method for laparoscopy. Am J Obstet Gynecol [Internet]. 1971 Jul 15 [cited 2021 Sep 21];110(6):886-7. Available from: http://www.ajog.org/article/00029378719059 3X/fulltext

7. Semm K, Semm I. Safe insertion of trocars and the Veress needle using standard equipment and the 11 security steps. Gynaecol Endosc [Internet]. 1999 Dec 1 [cited 2020 Dec 24];8(6):339-47. Available from: http://doi.wiley.com/10.1046/j.13652508.1999.00333.x

8. Veress J. Neues Instrument zur Ausführung von Brust-oder Bauchpunktionen und Pneumothoraxbehandlung. DMW-Deutsche Medizinische Wochenschrift. 1938;64(41):1480-1.

9. Ahmad G, Baker J, Finnerty J, Phillips K, Watson A. Laparoscopic entry techniques. Cochrane Database Syst Rev [Internet]. 2019 Jan 18 [cited 2020 Dec 24];2019(1). Available from: /pmc/articles/PMC6353066/?report=abstract

10. Lajer H, Widecrantz S, Heisterberg L. Hernias in trocar ports following abdominal laparoscopy: A review. Acta Obstet Gynecol Scand [Internet]. 1997 Jan 1 [cited 2020 Dec 24];76(5):389-93. Available from: http://doi.wiley.com/10.3109/000163497090 47816

11. Molloy D, Kaloo PD, Cooper M, Nguyen T V. Laparoscopic entry: A literature review and analysis of techniques and complications of primary port entry [Internet]. Vol. 42, Australian and New Zealand Journal of Obstetrics and Gynaecology. Royal Australian and New Zealand 

$\mathrm{KF}$, Shih TY, et al. Major complications of operative gynecologic laparoscopy in Southern Taiwan: A follow-up study. J Minim Invasive Gynecol. 2007 May 1;14(3):284-92.

21.

Fuller J, Ashar BS, Carey-Corrado J. Trocar-associated injuries and fatalities: An analysis of 1399 reports to the FDA. J Minim Invasive Gynecol [Internet]. 2005 Aug 1 [cited 2021 Sep 23];12(4):302-7. Available from: http://www.jmig.org/article/S1553465005002 979/fulltext

22. Merlin TL, Hiller JE, Maddern GJ, Jamieson GG, Brown AR, Kolbe A. Systematic review of the safety and effectiveness of methods used to establish pneumoperitoneum in laparoscopic surgery. Br J Surg [Internet]. 2003 Jun 9 [cited 2021 Sep 23];90(6):668-79. Available from: https://academic.oup.com/bjs/article/90/6/6 68/6143325

$23 . \quad$ Pring C. Aortic injury using the Hasson trocar: a case report and review of the literature. Ann R Coll Surg Engl [Internet]. 2007 Mar 1;89(2):3-5. Available from: http://openurl.ingenta.com/content/xref?genr e $=$ article\&issn $=0035$ -

8843\&volume $=89 \&$ issue $=2 \&$ spage $=3$

$24 . \quad$ Bhattacharya S, Tate JJT, Davidson BR, Hobbs KEF. Abdominal wall Haematoma Complicating Laparoscopic Cholecystectomy. HPB Surg. 1994;7(4):291-6.

25. Mayol J, Garcia-Aguilar J, Ortiz-Oshiro E, De-Diego Carmona JA, Fernandez-Represa JA. Risks of the Minimal Access Approach for Laparoscopic Surgery: Multivariate Analysis of Morbidity Related to Umbilical Trocar Insertion. World J Surg 1997215 [Internet]. 1997 Jun [cited 2021 Sep 23];21(5):529-33. Available from:

https://link.springer.com/article/10.1007/PL0 0012281 